\title{
Modelizando práticas para a socialização de informações: a construção de saberes no ensino superior
}

\section{Leilah Santiago Bufrem}

Doutora em Ciências da Comunicação pela Universidade de São Paulo, Pós-Doutora pela Universidad Autonoma de Madrid. Professora Titular do Departamento de Ciência e Gestão da Informação da Universidade Federal do Paraná, Curitiba, Paraná, Brasil

Francisco Daniel de Oliveira Costa

Aluno do curso de Gestão da Informação da Universidade Federal do Paraná. Bolsista de Iniciação Científica (PIBIC/CNPq)

Rene Faustino Gabriel Junior

Bacharel em Biblioteconomia e Documentação pela Pontifícia Universidade Católica do Paraná. Mestrando do programa Ciência, Gestão e Tecnologia da Informação na Universidade Federal do Paraná-UFPR

José Simão de Paula Pinto

Doutor em Medicina pela Universidade Federal do Paraná. Coordenador do Programa de pósgraduação em Ciência, Gestão e Tecnologia da Informação . Professor Adjunto do Departamento de Ciência e Gestão da Informação da Universidade Federal do Paraná

Propõe uma metodologia para a criação de ambiente integrado de monitoramento e gerenciamento de publicações periódicas para composição de dados da Base de Dados Referencial de Artigos de Periódicos em Ciência da Informação (Brapci). Define e analisa variáveis orientadoras da pesquisa e, por meio de um estudo exploratório e mapeamento das demandas dos usuários, realiza a testagem de um protótipo para a concretização da proposta. Descreve o planejamento, construção, implementação e validação do produto funcional do sistema, utilizando a rede internet como plataforma de aplicação. 
Palavras-chave: Bases de dados; Compartilhamento da informação; Arquitetura da informação; Brapci.

\section{Standardizing practices for socializing information: the building of knowledge in higher education}

This paper proposes a methodology to create an integrated environment monitoring for the management and publishing of the Referential Data Base of Articles from Information Science Periodicals (Brapci). Defines and examines variables guiding the research and, through an exploratory study and mapping of users demands, makes the accomplishment of a prototype for implementing the proposal. It describes the planning, construction, development and validation of the functional system using the Internet as a network application platform.

Keywords: Data Base; Information sharing; Information architecture; Brapci.

Recebido em 01.04.2010 Aceito em 30.06.2010

\section{Introdução}

O formato e a rapidez no acesso às informações têm sido influenciados pela Internet, fenômeno que transformou os modos de produção e recuperação dos produtos do conhecimento. A plataforma tecnológica da Word Wide Web tornou onipresente uma entidade antes rara: o ambiente de informações compartilhadas, concretizando o que tem sido denominado de site ou sítio.

No intuito de resgatar princípios voltados ao ideal da socialização da informação, modelos de organização e pesquisas operacionais têm sido desenvolvidos, resultando em expressões de esforços teórico-práticos em áreas para as quais a abordagem transdisciplinar tem sido efetivamente uma vantajosa estratégia. Uma dessas áreas é a Ciência da Informação (CI), especialmente quando se reúnem vertentes advindas de projetos voltados à produção, organização e utilização da informação científica para a criação de conhecimentos.

A organização das modalidades mais adequadas voltadas a esses propósitos é efetivada por um domínio hoje muito alentado, a arquitetura da informação que, segundo Fox (2001), é a arte e a ciência de estruturar e organizar sistemas para auxiliar as pessoas a alcançarem seus objetivos na busca informacional. As etapas para o desenvolvimento da arquitetura 
da informação, contudo, requerem a elaboração de uma estratégia que abrange análise do conteúdo, testes com os usuários e opiniões resultantes de entrevistas, frente às dificuldades de se organizar sites com excesso de informações. Hylton (2000), em uma revisão de literatura sobre o tema, destaca que a qualidade no uso de um site pode ser medida pelo comportamento e satisfação do usuário no momento do acesso e no resultado da busca pela informação esperada.

O trabalho de modelagem de informações atende à exigência de torná-las relevantes e oportunas, requerendo estudo especializado. A implantação desses modelos, por meio de informações acessíveis e compartilhadas de modo universal, é um tipo específico de atividade relativamente recente, que consiste tanto em aplicação científica de saberes específicos quanto em atividade artística, um ato de arquitetura, estruturando informações cruas em ambientes compartilhados de forma útil, navegável e funcional às reais necessidades, de modo a resistir à entropia e reduzir a confusão.

No Brasil, atualmente, alguns títulos de revistas científicas encontram-se disponíveis para consulta em sites de acesso livre, mas ainda são insuficientes por abrangerem apenas as revistas eletrônicas e/ou serem disponibilizados on-line. Daí a necessidade sentida de se criar uma base de dados referenciais de revistas nacionais da área de $\mathrm{CI}$, reunindo a literatura científica impressa e eletrônica a ela relacionada e possibilitando estudos quantitativos e qualitativos sobre a produção editorial da área.

Os estudos sobre revistas científicas na literatura recente, mais especificamente nos dez últimos anos, embora com marcante presença dos dados quantitativos como base empírica para reforçar argumentações, revelam uma tendência à análise e interpretação de caráter qualitativo, especialmente justificada pela complexidade de fatores intervenientes nas atividades de produção e divulgação científicas.

Contribuições de Unger e Freire (2006) para este estudo partem de uma visão da relevância da informação na sociedade contemporânea e do propósito de organizar estruturas que alcancem os possíveis usuários. Assim, profissionais da informação devem considerar para o desenvolvimento de suas atividades: o contexto sócio-econômico-cultural em que se inserem o agregado e seus estoques de informação e o grupo de usuários que Ihes interessa; um modelo de sistema de informação que atenda às características desses usuários potenciais e uma linguagem documentária, que melhor represente o conhecimento oculto nos estoques de informação, de modo a diminuir as barreiras na comunicação entre o sistema e seus usuários. Ao permear o processo de planejamento de uma base de dados, a presença do usuário impõe-se para a definição dos propósitos e como orientação para a avaliação dos processos e do produto gerado. O corpus deve ser, portanto, representativo das necessidades reais e potenciais da comunidade usuária. 
A Base de Dados Referencial de Artigos de Periódicos em Ciência da Informação (Brapci ${ }^{1}$ ) é o produto de informação do projeto de pesquisa "Opções metodológicas em pesquisa: a contribuição da área da informação para a produção de saberes no ensino superior", cujo objetivo tem sido subsidiar estudos e propostas na área de CI, fundamentando-se em atividades planejadas institucionalmente. Com esse propósito, são identificados os títulos de periódicos da área de CI e indexados seus artigos, constituindo-se o corpus da base de dados referenciais. Inicialmente ambientada no ProCite 5, a Base tem catalogado referências e resumos de textos publicados em periódicos nacionais impressos e eletrônicos da área de CI a partir de 1972.

Desde sua concepção até o desenvolvimento de uma versão funcional, a Brapci está contribuindo para estudos analíticos e descritivos sobre a produção editorial de uma área em desenvolvimento, ao subsidiar com uma ferramenta dinâmica estudantes, professores e pesquisadores não somente da área de $\mathrm{CI}$, mas também de outras a ela relacionadas. Além disso, integra pesquisadores e estudantes de graduação e pósgraduação na construção de saberes relacionados às áreas que contribuem para o processo de pesquisa requerido para sua concretização.

O objetivo deste estudo é o desenvolvimento de um produto final, visando a disponibilização dos dados da Brapci para acesso público, utilizando-se a Internet como meio disseminador e ferramentas que possibilitem sua manutenção e operacionalização. Para efetivação desse propósito mais amplo, foram desmembrados objetivos específicos: 0 planejamento do conteúdo textual do sistema, partindo dos resultados do mapeamento e da pesquisa de campo; a classificação, separação e hierarquização das sessões do sistema; a elaboração do projeto visual, definindo-se tipologia, iconografia e padrões gráficos e de cores do sistema; o estudo e definição das opções de linguagens e ferramentas operacionais apropriadas para a execução do produto; o desenvolvimento e programação do produto; a validação do produto, com a realização de pesquisa de campo instrumentalizada por grupo de foco composto por pesquisadores da área; e a realização, com base na validação, das considerações finais e modificações no produto e metodologia desenvolvidos. O ambiente específico de informações compartilhadas, integrando usuários e pesquisadores, adquiriu caráter inovador ao resultar em uma superfície ergonômica, acessível, usável e facilmente recuperável. Seus processos foram observados e devidamente documentados. Para a adaptação aos diferentes perfis de usuários de uma base com essas características, o delineamento da máscara ou espelho que comportou os itens e as interfaces pertinentes aos perfis foram objetos de constante atualização, exatidão, conformação de estilo apropriado ao conteúdo e adaptação aos usos.

Dessa forma, além de seu propósito pedagógico, como o estímulo à investigação científica pelo grupo de estudantes de graduação e pós-

\footnotetext{
${ }^{1}$ BASE REFERENCIAL DE ARTIGOS DE PERIÓDICOS EM CIÊNCIA DA INFORMAÇÃO. Curitiba: UFPR, 2009. Disponível em: <www.brapci.ufpr.br>. Acesso em 30 out. 2009.
} 
graduação que compõem a equipe de pesquisa, ampliaram-se, com este estudo, as possibilidades de análise e interpretação das informações constantes em bases de dados com vistas à ampliação da confiabilidade dos resultados de busca.

\section{Referencial teórico}

O vasto universo de saberes registrados, no qual se destacam as revistas científicas, oferece perspectivas para a compreensão da história da produção intelectual de áreas específicas, especialmente quando analisado seu conteúdo sob uma visão diacrônica. A experiência enseja a reflexão sobre conteúdos, categorias, linhas, enfoques e métodos utilizados nas pesquisas (BUFREM, 2006).

Esse contexto e as repercussões dos avanços científicos sobre as formas de produção do conhecimento são comentados por Ladrière (1978, p. 10), para quem a ciência age sobre a realidade, transformando-a, sobretudo, por meio da tecnologia, face visível de suas produções. Impõese, todavia, a tarefa crítica à prática no sentido de se procurarem formas coerentes de intervenção na realidade, especialmente em programas de formação acadêmica. Se a pesquisa pode ser considerada um meio de conhecimento integrador de teoria e prática, o seu aperfeiçoamento é a razão pela qual acadêmicos e profissionais vêm tomando consciência da necessidade de ampliar a compreensão a respeito das possibilidades teóricas e concretas ao seu alcance para avaliar e aperfeiçoar suas formas de aquisição do saber.

Essa tem sido a razão pela qual pesquisadores vêm procurando ampliar sua compreensão sobre as possibilidades e formatos de produção e representação do texto científico. O estudo justifica-se, sobremodo, no universo da produção do saber nas instituições de ensino superior, vasto e estimulante em sua complexidade, graças aos desafios das matérias do conhecimento que se desdobram em suas problemáticas e aos modos de apreensão dessas matérias, como que a confirmar que ao saber científico não se podem estabelecer fronteiras. Ao discutir a necessidade de avaliação prévia e amadurecimento nas ideias pioneiras de democratização na publicação do conhecimento científico e avanço na sua aceitação, Mueller (2006) reconhece também o papel das editoras e das elites de cada área, bem como as relações de poder e influência na direção e velocidade do percurso das publicações eletrônicas de acesso livre e sua incorporação ao sistema de comunicação científica, como canais legítimos desse processo.

Ao definir as bases de dados como repositórios dos conhecimentos consensuais gerados pela ciência moderna, Sayão (1996) as considera constituintes da memória da ciência oficialmente aceita. Sua existência justifica-se especialmente pela necessidade que os pesquisadores e estudantes têm de informações sobre as fontes disponíveis para o domínio, sempre relativo, da literatura de sua área e dos meios existentes 
para difusão de seus próprios estudos. Além disso, a publicação científica tornou-se, em seu processo histórico, um instrumento indispensável tanto como meio de promoção acadêmica quanto como modalidade de promoção e fortalecimento do ciclo criação, organização e difusão do conhecimento. Por conseguinte, sua divulgação é um dos fatores que mais influenciam a realização deste estudo.

É indiscutível a importância das bases de dados na realização de pesquisas quantitativas e qualitativas, ao facilitarem a rápida localização e análise da informação nelas contida. Além disso, a possibilidade da avaliação feita pelos pares é processo indispensável, segundo Meadows (1999), para que se obtenham o consenso dos pesquisadores e a consequente credibilidade quanto aos resultados da pesquisa comunicada. Com a crescente adoção das tecnologias de informação, pode-se considerar uma base de dados uma fonte que, segundo Dias (2000), indica aquelas obras de uso pontual e recorrente. Seu uso tem se tornado cada vez mais comum devido às vantagens que oferece em relação aos índices impressos, especialmente como instrumento de recuperação de informação que permite realizar pesquisas complexas, o que se torna inviável nos instrumentos impressos convencionais. Todas essas facilidades representam uma grande economia de tempo para o usuário, permitindo que uma pesquisa seja executada bem mais rapidamente com o uso dos computadores (CENDón, 2000).

O trabalho de adaptação e aperfeiçoamento contínuo de uma base requer a consideração de indicadores, tais como: os dispositivos de saída dos dados, as informações disponibilizadas, a visualização da informação, o retorno possível do usuário, o comportamento do sistema e os sistemas de apoio à base. É precisamente na comunicação e na necessidade de que ambas as partes utilizem os mesmos códigos que reside a chave do arco de êxito ou fracasso da interação, segundo García López (2007).

Nesse processo, a visualização implica mais do que simplesmente o ato de olhar, segundo concepção de García López (2007), a partir de Dürsteler (2002), uma vez que é uma construção mental, próxima do conhecimento e, portanto, uma apreensão intelectual. Se o entendimento significa a contextualização, inclusão e interiorização de algo é um ato que se modifica e modifica a estrutura nocional dos sujeitos. A qualidade de uma base de dados é, portanto, fator de estímulo, conducente e mobilizador desse entendimento, para o qual contribuem metáforas visuais, mapas, muros de perspectivas ou árvores de representações hierárquicas ou em rede, mapas de dispersão, lentes e browser, com os focos que permitem.

Nenhuma dessas formas, entretanto, dispensa, quando se projeta a qualidade na relação ser humano/máquina, a recorrência a técnicas de transformação visual, como propõem Gutwin e Fedak (2004 apud GARCÍA LÓPEZ, 2007).

Outras virtudes decorrentes desse propósito mais amplo referem-se ao uso concreto propiciado pela modalidade de apresentação e atualização 
dos dados. Nesse sentido, a arquitetura de uma base de dados é efetiva para a integração entre técnicas e propósitos.

Ao argumentarem que "uma arquitetura bem elaborada pode permitir uma interação mais rápida e fácil entre o usuário e a informação", Camargo e Vidotti (2006, p. 105) justificam que a arquitetura da informação, de um modo geral, unifica os métodos de organização, classificação e recuperação de informação com a exibição espacial da área de arquitetura, utilizando-se de tecnologias de informação e comunicação e, em especial, da Internet.

A arquitetura da informação envolve quatro elementos básicos, que visam à criação de estruturas digitais, nas quais priorizam a organização descritiva, temática, representacional, visual e navegacional de informações (ROSENFELD; MORVILLE, 2002):

a-sistemas de organização, responsáveis pela estruturação e formas de agrupamentos do conteúdo do site;

b-sistemas de rotulagem, responsáveis pela denominação do conteúdo do grupo informacional, agindo na representação ou identificação de conteúdos específicos;

c-sistemas de navegação, responsáveis pela apresentação de pontos de referência ao usuário, por meio de barras de navegação e mapas do site;

d-sistemas de busca, responsáveis pelo auxílio ao usuário nas consultas, inclusive prever as buscas que o usuário pode fazer e 0 conjunto de respostas possíveis.

Esse processo pode ser complementado pela usabilidade que, segundo Nielsen (1993), não se trata de um requisito único na elaboração de uma interface para usuários, visto que consiste em cinco atributos básicos: facilidade de aprendizado (learnability); efetividade de uso (efficiency); de fácil memorização (memorability); minimização de possibilidades de erro (errors); e satisfação do usuário durante a navegação (satisfaction). O único meio de se avaliar a qualidade da usabilidade é através de testes com os reais usuários durante o processo de navegação.

Ao tratar dos requisitos de usabilidade, Santos (2006) os considera fatores responsáveis pela qualidade da interação do usuário com o aplicativo. Tais conceitos são importantíssimos em aplicações Internet, já que as mesmas estão "sempre disponíveis e em constante crescimento" (BROWN, 2003, p. 188; YU, 2005, p. 220).

Essas e outras leituras evidenciaram a importância de um projeto de arquitetura da informação voltado à disponibilização da Brapci na Web, não só para contribuir com o processo de indexação, mas, principalmente, com a qualidade do acesso pelo usuário à base referencial, de modo a facilitar a recuperação e o entendimento da informação desejada.

As contribuições da literatura, sem dúvida, esclarecem aspectos da prática concreta relacionada às fases de planejamento, construção, atualização, padronização, disponibilização e utilização de bases de dados. 
Entretanto, por mais atuais e abrangentes que sejam seus ensinamentos, indicam a complexidade das estruturas que suportam esse tipo de realização.

\section{Metodologia}

A continuidade do levantamento bibliográfico é fundamental para uma atualização permanente sobre as mais recentes aquisições desse domínio. Foram realizados estudos e análises sobre softwares já existentes de auxílio à construção de documentos científicos, citação bibliográfica e recuperação de referências, como medida de benchmarking.

Por meio da análise dos dados disponíveis na Brapci e do material de pesquisa já produzido pela equipe do projeto, foram coletadas informações sobre a base, suas vertentes e sua estrutura de armazenamento para a análise dos requisitos funcionais relacionados à base e às equipes de todo o projeto de pesquisa. O diagnóstico foi complementado com discussões envolvendo os pesquisadores deste projeto e professores dos cursos de graduação em Gestão da Informação da UFPR.

O estudo exploratório foi fundamental para a definição das variáveis orientadoras da pesquisa de campo e englobou as atividades relacionadas à leitura e à observação das discussões.

A pesquisa de campo para mapeamento das demandas dos usuários foi realizada a partir da lista de discussão da Ancib. Foram distribuídos eletronicamente questionários para todos os membros da lista e estabelecido prazo de um mês para retorno. Por ter sido utilizada uma lista de discussão eletrônica não foi possível definir o universo dos pesquisadores, mas no prazo estabelecido retornaram 47 questionários. Para codificação e tabulação dos dados foi utilizada uma planilha do MSExcel como meio de identificar as necessidades informacionais dos pesquisadores.

A análise e a interpretação dos dados fundamentaram-se na literatura pertinente e suas considerações aliadas ao mapeamento das necessidades, os resultados e as observações das leituras realizadas, que serviram como base para a definição dos requisitos e indicadores de qualidade do produto.

Com a coleta de dados concluída, iniciou-se a fase de modelagem do produto, constituída pelo planejamento do conteúdo textual do sistema, utilizando-se os resultados do mapeamento das análises e das definições dos requisitos e indicadores levantados empiricamente. Foram então classificadas, separadas e hierarquizadas as sessões do sistema. Na fase de projeto, as abordagens sugeridas por Yu (2005) referem-se à 
estrutura; ambiente de desenvolvimento; e ferramentas, tipos de arquivos, linguagens e padrões.

Para elaboração do projeto visual definiram-se a tipologia, a iconografia e os padrões gráficos e de cores do sistema por meio de estudo da literatura e de casos similares. Da mesma forma, as opções de linguagens e ferramentas operacionais apropriadas para a execução do produto foram estudadas e definidas pela literatura, assim como as limitações e configurações técnicas oferecidas pelo Centro de Computação Eletrônica (CCE) da Universidade Federal do Paraná (UFPR). Da linguagem escolhida para o produto e o sistema gerenciador de banco de dados (SGBD) foram utilizados respectivamente um servidor Apache ${ }^{1}$ com suporte ao PHP 2 e o MySQL, por utilizarem licença livre. Analisaram-se os fluxos de navegação possíveis, para uma avaliação qualitativa e de usabilidade de cada fase do sistema.

O desenvolvimento e a implantação do produto foram realizados de forma restrita ao grupo de foco selecionado com o objetivo de avaliá-lo, constituído de pesquisadores bolsistas de Iniciação Científica e estudantes de graduação e pós-graduação da UFPR. Procurou-se, por meio dessa técnica, identificar a percepção do grupo quanto à utilização e aplicação da Brapci. Dessa forma, foram previstas questões que permitiram aos participantes do grupo, com auxílio de um moderador, comentar as questões levantadas (KRUEGER; CASEY, 2000). O produto do sistema foi desenvolvido pelo grupo, com suporte técnico de infraestrutura do CCE da UFPR, para a realização das sessões. Realizou-se na sequência um estudo qualitativo das informações para disponibilização irrestrita, livre e universal da base on-line pela Internet.

Os processos de criação, manutenção e organização de produtos voltados ao inventário das produções científicas têm sido uma preocupação crescente da sociedade, proporcional ao crescimento dos registros do conhecimento e ao consequente volume das informações. Produtos, serviços e estruturas gigantescas são gerados com potencial de armazenamento, gerenciamento e disponibilidade. Nesse cenário de inovações, os profissionais da informação buscam especializações, modelos e parâmetros de usabilidade e pertinência.

Entretanto, o simples desenvolvimento de um sistema gerenciador, sem uma arquitetura das informações com indicadores de qualidade em seu planejamento, pode resultar em um produto final inadequado. A utilização de ferramentas que ofereçam estruturas lógicas e induzam à respostas satisfatórias em relação aos objetivos do usuário salientam-se como contraponto àquelas que não apresentam a mínima organização e frustram as necessidades dos seus utilizadores (ROSENFELD; MORVILLE, 2002). O projeto adequado é importante, pois o ambiente proporciona ao usuário interação com a informação. Na FIG. 1 vê-se que a informação para interação é gerada a partir do conteúdo (1), gerando opções ao usuário (2), que as seleciona (3), gerando uma busca (4) e entrega (5) do

\footnotetext{
${ }_{1}$ Servidor Apache, um software livre de servidor para web. Disponível em: <http://www.apache.org/>. Acesso em: 30 out. 2009.
} 
Modelizando práticas para a socialização de informações: a construção de saberes no ensino superior
Leilah Santiago Bufrem; Francisco Daniel de Oliveira Costa; René Faustino Gabriel Junior; José Simão de Paula Pinto

conteúdo solicitado. Embora simples, o modelo define a interação usuário/servidor por meio do ambiente web (interação, seleção e entrega).

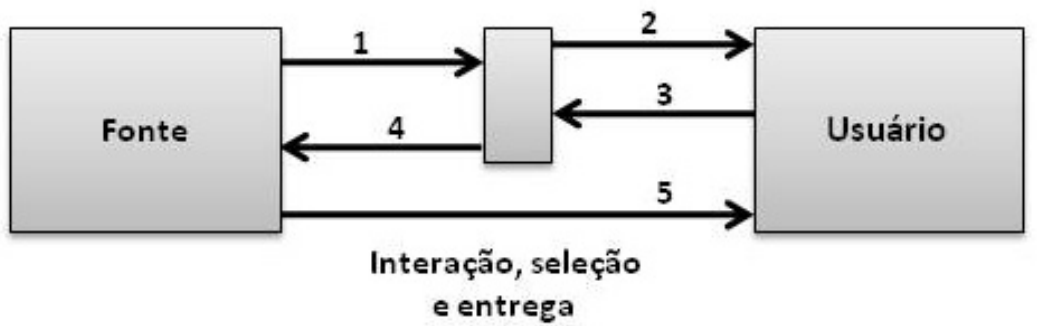

FIGURA 1 - Cenário para entrega de informações

Fonte: Adaptado de BROWN (2003, p. 24).

O estudo defende a utilização desses conceitos, agregados às competências do Gestor da Informação, para a concepção e a implantação de um sistema desde a criação do banco de dados, ambiente gráfico e funcionalidades até a satisfação do usuário com os resultados obtidos no acesso e utilização das funcionalidades oferecidas.

Para atingir o propósito mais amplo desta pesquisa, foram estruturados os elementos com vistas a um modelo conceitual de um produto funcional do sistema de gerenciamento e publicação dos dados da Brapci. Visando sua concepção e implantação, utilizou-se na metodologia a estrutura advinda da teoria de desenvolvimento de produtos segundo Rozenfeld e colaboradores (2006), de acordo com a FIG. 2.

\begin{tabular}{|l|l|l|l|}
\hline \multicolumn{1}{|c|}{ Pré } \\
\hline $\begin{array}{l}\text { Planejamento } \\
\text { Estratégico do } \\
\text { Produto }\end{array}$
\end{tabular}

FIGURA 2 - Fases do desenvolvimento do produto 
Fonte: Adaptado de ROZENFELD et. al (2006).

Para a concepção da Brapci, a partir dos resultados do mapeamento e dados levantados na primeira fase do projeto, realizou-se o planejamento do conteúdo textual e funcionalidades do sistema, por meio de estudo exploratório para determinar perfis de usuários e fluxos de trabalho na base. Foram utilizados os conceitos da arquitetura da informação para definir a relevância de cada item, suas instâncias para cada grupo de usuários, a estrutura da informação e a prioridade de apresentação dessas informações. Desse modo, realizou-se a modelagem de dados por meio de sua decomposição e da metodologia formal para avaliação de um esquema relacional, conforme proposta de Silberschatz, Korth e Sudarshan (2006). A interface do sistema proposto foi projetada por meio da construção de wireframes. Todas as etapas do processo foram acompanhadas paralelamente por discussões entre os integrantes do Grupo de Pesquisa, o que favoreceu o aperfeiçoamento contínuo no produto e na metodologia desenvolvidos.

A partir da análise do fluxo de trabalho, foram definidos os perfis de usuários da base e mapeamento das funções do sistema (QUADRO 1). Categorizadas as permissões de uso, elaborou-se um novo processo para indexação e manutenção da Brapci com a possibilidade de ocorrerem atividades simultâneas e on-line diretamente no sistema (FIG. 3).

QUADRO 1 Atividades permitidas a cada perfil de usuário

\begin{tabular}{|c|c|c|}
\hline \multicolumn{2}{|l|}{ Atividade } & \multirow{2}{*}{$\begin{array}{c}\text { Perfil autorizado a realizar } \\
\text { Coordenador }\end{array}$} \\
\hline Indexar novo Periódico & & \\
\hline Indexar novas Edições & & Editor / Indexador \\
\hline Indexar novos Artigos & & Todos \\
\hline Realizar modificações & nos & Coordenador \\
\hline \multicolumn{3}{|l|}{ Periódicos } \\
\hline Realizar modificações & nas & Editor \\
\hline \multicolumn{3}{|l|}{ Edições } \\
\hline Realizar modificações & nos & Editor / Indexador \\
\hline \multicolumn{3}{|l|}{ Artigos } \\
\hline Excluir Periódicos & & Nenhum* \\
\hline Excluir Edições & & Nenhum* \\
\hline Excluir Artigos & & Editor \\
\hline Autorizar/Suspender & & Coordenador / Editor \\
\hline \multicolumn{3}{|l|}{ Publicação da Edição } \\
\hline Gerar Relatórios & & Todos \\
\hline Criar Usuários & & Coordenador / Editor \\
\hline
\end{tabular}


Nota: $\left({ }^{\star}\right)$ A exclusão de Periódicos e Edições só é permitida ao administrador do banco de dados da Brapci para que não ocorra perda de integridade relacional dos dados.

Fonte: COSTA, 2009.

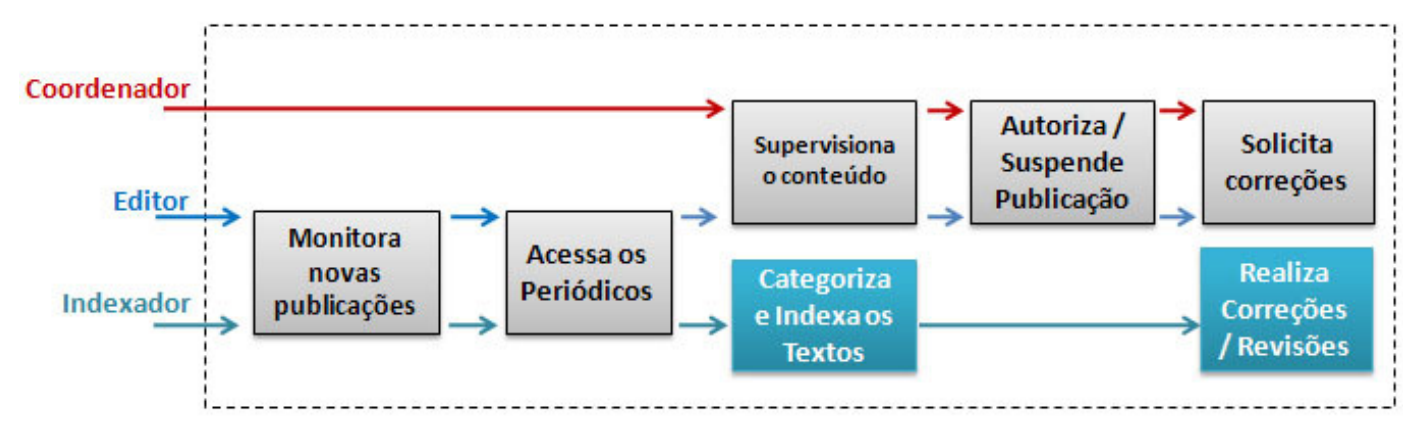

FIGURA 3 - Processo proposto para indexação e manutenção da BRAPCI Fonte: COSTA, 2009.

Com a definição dos perfis e das atividades que cada usuário pode realizar na Base, partiu-se para o processo de migração da base de dados Brapci para uma nova modelagem de dados, demandando a decomposição de uma tabela única (do ProCite5) para múltiplas tabelas, utilizando-se um SGBD, por meio da metodologia proposta por Silberschatz, Korth e Sudarshan (2006), pela qual todas as dependências funcionais devem estar relacionadas exclusivamente à chave primária.

Nessa modelagem de dados, priorizou-se a utilização de várias tabelas relacionais, partindo-se do Periódico (Revista) e desmembrandoas em outras tabelas, técnica que facilita a normatização dos registros, pois é criado um padrão que consequentemente contribui para a qualidade da base de dados. Buscou-se, com a modelização dos dados, uma especificidade que possibilitasse a identificação da autoria, sua titulação e instituição afiliada, tipo de seção publicada, edições, palavras-chave em diversos idiomas e metodologias dos trabalhos. Essa modelização permitiu a seleção de dados para análise de conteúdo, análise de produção por autor, titulação e outras características, conforme as necessidades do pesquisador (FIG. 4) 


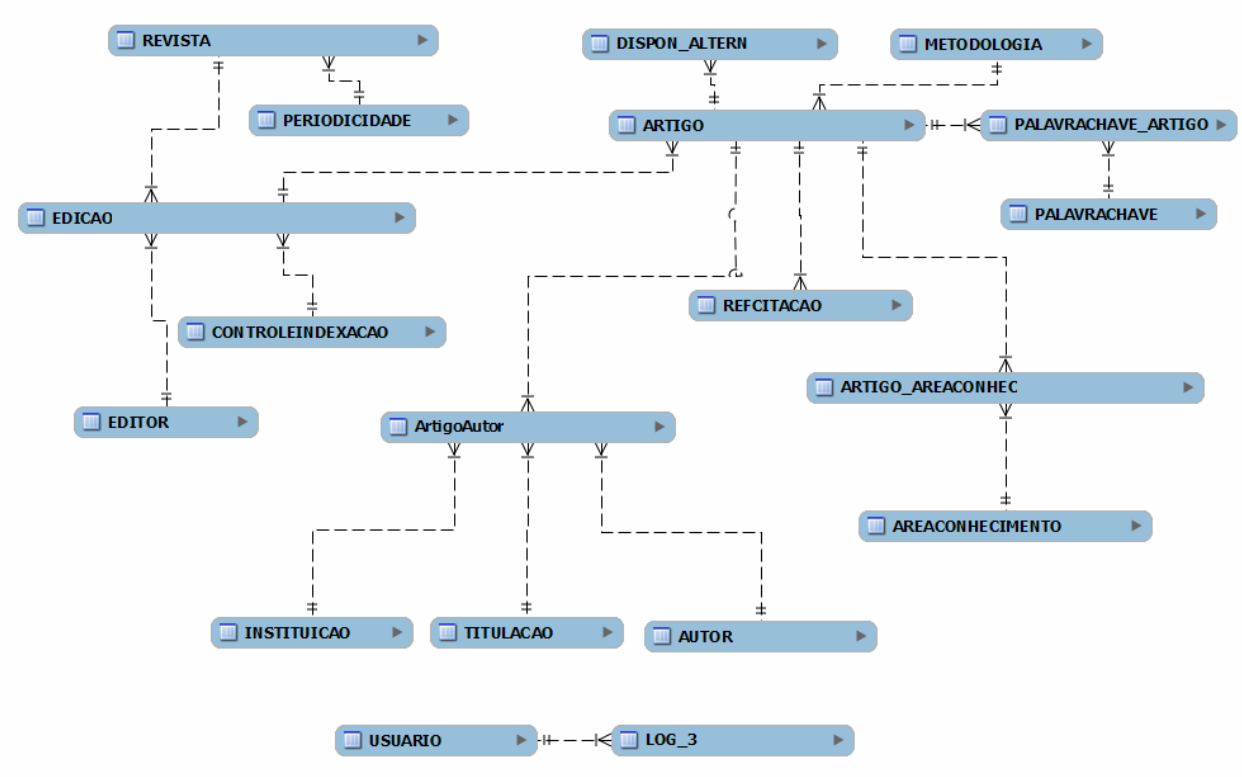

FIGURA 4 - Diagrama entidade-relacionamento do novo modelo de banco de dados

Fonte: Os autores (2009).

Utilizando-se os conceitos de arquitetura da informação levantados e definidos os requisitos da base de dados, foi elaborada a modelagem conceitual de sua interface sob a forma de wireframes.

O desenvolvimento do produto físico foi acompanhado pela coordenação do projeto e, após sua finalização e implantação, avaliado pelos usuários. A detecção e correção de falhas e inovações, cujas consequências são vitais para o aprimoramento da tecnologia empregada, estão sendo realizadas para o aperfeiçoamento do novo sistema pelo Grupo.

A nova estrutura possibilitou, ainda, a integração através do protocolo Open Archives Iniciative Protocol for Metadata Harvesting (OAI$\mathrm{PMH})^{3}$, utilizado pelo SEER com revistas e periódicos nacionais, otimizando 0 processo de indexação e permitindo o aumento da abrangência do corpus da Base por meio de coletas periódicas. Desse modo, há a possibilidade de transferência desses conhecimentos para outros domínios, respeitadas as especificações e peculiaridades que os conformam.

Como mérito principal dessa realização, os resultados esperados atingem o ideal universitário da integração ensino-pesquisa-extensão, em um verdadeiro processo pedagógico de construção e socialização de saberes.

\footnotetext{
${ }^{2}$ OAl-PMH (Open Archives Initiative Protocol for Metadata Harvesting) é um protocolo desenvolvido pela Open Archives Initiative, utilizado para distribuir e coletar metadados relativos a descritores de documentos.
} 


\section{Um retrato da Brapci}

Desde a ideia inicial de reunir a literatura pertinente da área de CI em um único local que facilitasse a busca e recuperação da informação para pesquisadores, acadêmicos e a comunidade em geral já se passaram mais de dez anos. O resultado final na criação de um produto que possa ser acessado por todos em um ambiente virtual e coletivo é a concretização dessa ideia.

Nos três primeiros anos da implantação do projeto (2000-2003), foram levantados os títulos de treze periódicos da área e, paralelamente, realizada busca no acervo da Biblioteca do Setor de Ciências Sociais Aplicadas (BSCSA) da UFPR, onde foram verificados os periódicos do acervo físico relacionados à área de CI. Os fascículos não encontrados foram solicitados aos editores ou, após localização no Catálogo Coletivo Nacional de Publicações Seriadas (CCN)4, a outras bibliotecas, para que fossem preenchidos os dados de acordo com os campos definidos para a sua representação na base que inicialmente era desenvolvida no ProCite ${ }^{3}$.

Nos anos seguintes (2004-2008), foram realizadas análises específicas sobre as características temáticas, metodológicas e formais da literatura, obedecendo-se aos pressupostos iniciais e incorporação à base das publicações que atendiam a esses critérios. Essa análise possibilitou a ampliação significativa da quantidade de títulos selecionados; dos treze iniciais para 27 títulos até 2008, registrando-se 4.637 artigos publicados (BUFREM, 2008).

Em 2009, foram incorporados mais três títulos de publicações à Brapci e implementado o mecanismo de coleta automática de registros, utilizando-se o protocolo OAI-PMH de arquivos abertos. Isso possibilitou um crescimento significativo da base em quase dois mil registros adicionais, graças ao acesso em ambiente virtual5 de edições antes não acessíveis, as quais foram identificadas e incorporadas por meio de trocas de metadados.

Até outubro do ano de 2009, a Brapci reuniu trinta publicações periódicas vigentes e históricas produzidas no Brasil, concentrando 6167 artigos em CI. As publicações disponíveis na base estão relacionadas no QUADRO 2.

\footnotetext{
${ }^{3}$ O Catálogo Coletivo Nacional de Publicações Seriadas (CCN), coordenado pelo IBICT, é uma rede cooperativa de unidades de informação localizadas no Brasil com o objetivo de reunir, em um único Catálogo Nacional de acesso público, as informações sobre publicações periódicas técnico científicas reunidas em centenas de catálogos distribuídos nas diversas bibliotecas do país.
} 
Modelizando práticas para a socialização de informações: a construção de saberes no ensino superior
Leilah Santiago Bufrem; Francisco Daniel de Oliveira Costa; René Faustino Gabriel Junior; José Simão de Paula Pinto

QUADRO 2 Principais características e informações das revistas indexadas

\begin{tabular}{|c|c|c|c|c|}
\hline Revista & ISSN & Publicação & Artigos & Disponib. \\
\hline Arquivística.net & $1808-4826$ & Vigente & 56 & $\mathrm{E}$ \\
\hline Arquivo \& Administração & $0100-2244$ & Vigente & 60 & I \\
\hline $\begin{array}{l}\text { BIBLOS: Revista do Departamento de } \\
\text { Biblioteconomia e História }\end{array}$ & $0102-4388$ & Vigente & 349 & $\begin{array}{c}\mathrm{Ie} \\
\mathrm{E}\end{array}$ \\
\hline Cadernos de Biblioteconomia & $0102-6607$ & Histórica & 74 & I \\
\hline Ciência da Informação & $\begin{array}{l}0100-1965 \\
1518-8353^{*}\end{array}$ & * Vigente & 1035 & I\&E \\
\hline Comunicação \& Informação & $1415-5842$ & Vigente & 135 & 1 \\
\hline DataGramaZero & $1517-3801$ & Vigente & 184 & $\mathrm{E}$ \\
\hline $\begin{array}{l}\text { Em Questão: Revista da Faculdade de } \\
\text { Biblioteconomia e Comunicação da UFRGS }\end{array}$ & $\begin{array}{l}1807-8893 \\
1808-5245^{*}\end{array}$ & * Vigente & 229 & $\begin{array}{ll} \\
E\end{array}$ \\
\hline $\begin{array}{l}\text { Encontros Bibli: Revista Eletrônica de } \\
\text { Biblioteconomia e Ciência da Informação }\end{array}$ & $1518-2924$ & Vigente & 267 & $\mathrm{E}$ \\
\hline $\begin{array}{l}\text { Estudos Avançados em Biblioteconomia e Ciência } \\
\text { da Informação }\end{array}$ & 0100-9869 & Histórica & 23 & $\mathrm{I}$ \\
\hline ETD - Educação Temática Digital & $\begin{array}{l}1517-2539 \\
1676-2592 \\
\text { a partir } \\
\text { dez. } 2005\end{array}$ & Vigente & 369 & $\mathrm{E}$ \\
\hline Inclusão Social & $1808-8678$ & Vigente & 64 & $E$ \\
\hline Infociência & $1415-0018$ & Vigente & 13 & I \\
\hline Informação \& Informação & $1414-2139$ & Vigente & 202 & I\&E \\
\hline Informação \& Sociedade: Estudos & $\begin{array}{l}0104-0146 \\
1809-4783^{*}\end{array}$ & Vigente & 452 & $\mathrm{E}$ \\
\hline $\begin{array}{l}\text { Informare: Cadernos do Programa de Pós- } \\
\text { Graduação em Ciência da Informação }\end{array}$ & 0104-9461 & Histórica & 61 & \\
\hline Liinc em revista & $1808-3536$ & Vigente & 66 & $E$ \\
\hline Perspectivas em Ciência da Informação & $1413-9936$ & Vigente & 370 & $\mathrm{I}$ \\
\hline Revista ACB: Biblioteconomia em Santa Catarina & $1414-0594$ & Vigente & 399 & I\&E \\
\hline $\begin{array}{l}\text { Revista Brasileira de Biblioteconomia e } \\
\text { Documentação }\end{array}$ & $\begin{array}{l}0100-0691 \\
1980-6949^{*}\end{array}$ & Vigente & 284 & I\&E \\
\hline Revista da Escola de Biblioteconomia da UFMG & $0100-0829$ & $\begin{array}{l}\text { Histórica. Mudou } \\
\text { nome }\end{array}$ & 309 & I \\
\hline Revista de Biblioteconomia \& Comunicação & $0103-0361$ & $\begin{array}{l}\text { Histórica. Mudou } \\
\text { nome }\end{array}$ & 102 & $\mathrm{I}$ \\
\hline Revista de Biblioteconomia de Brasília & $0100-7157$ & Vigente & 562 & I\&E \\
\hline $\begin{array}{l}\text { Revista Digital de Biblioteconomia \& Ciência da } \\
\text { Informação }\end{array}$ & 1678-765X & Vigente & 147 & $\mathrm{E}$ \\
\hline $\begin{array}{l}\text { Revista do Departamento de Biblioteconomia e } \\
\text { História }\end{array}$ & 0101-045X & $\begin{array}{l}\text { Histórica. Mudou } \\
\text { nome }\end{array}$ & 9 & I \\
\hline Revista Eletrônica Informação e Cognição & $1807-8281$ & Vigente & 50 & $E$ \\
\hline Revista On-line da Biblioteca Prof. Joel Martins & 1517-3992 & $\begin{array}{l}\text { Histórica. Mudou } \\
\text { nome }\end{array}$ & 59 & $\mathrm{E}$ \\
\hline Revista Ponto de Acesso & $1981-6766$ & Vigente & 68 & I\&E \\
\hline $\begin{array}{l}\text { Tendências da Pesquisa Brasileira em Ciência da } \\
\text { Informação }\end{array}$ & $1983-5116$ & Vigente & 17 & $\mathrm{E}$ \\
\hline \multirow[t]{2}{*}{ Transinformação } & 0103-3786 & Vigente & 352 & I\&E \\
\hline & & & 6467 & \\
\hline
\end{tabular}

Nota: $\left({ }^{*}\right)$ ISSN Eletrônico.

Fonte: Os autores (2009).

O crescimento da base tem se desenvolvido com o monitoramento das publicações e incorporações de novas edições pelos indexadores. Dos 6467 artigos disponibilizados na base, 70\% concentram-se em dez revistas (QUADRO 2). São vigentes vinte e três das revistas e sete delas estão na Base pelo valor histórico e as contribuições para a área.

O Gráfico 8 demonstra a produção nacional na área de CI pela quantidade de artigos publicados referentes ao ano de produção. Observa- 
se um crescimento significativo a partir de 1996 e outro em 2006 e, esse último, mantendo-se estável em 2007 e 2008. O ano base de 2009 registra uma produção de 262 artigos publicados, valor que não representa ainda um decréscimo das publicações, principalmente pelas dificuldades de manutenção da periodicidade das revistas.

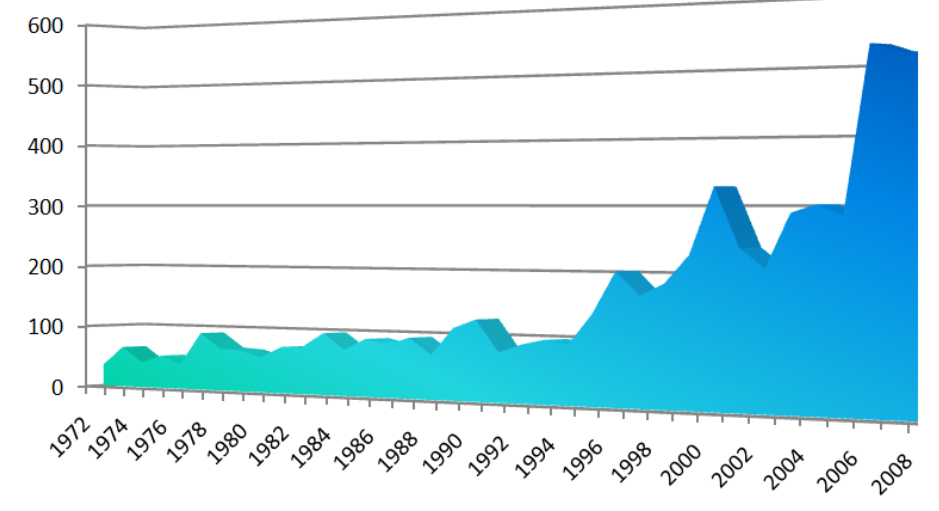

\section{GRÁFICO 1 - INSERÇÃO DE ARTIGOS NA BASE DE DADOS POR ANO DE PUBLICAÇÃO}

Fonte: Os autores (2009).

Com a disponibilização, em abril de 2008, de um módulo público com um mecanismo de busca simplificado foi possível abrir à comunidade acadêmica a consulta à Base. Considerando-se que toda a disponibilização, para que possa ser aperfeiçoada, necessita de uma monitoração e de um retorno de seus usuários com críticas e sugestões, todas as consultas realizadas na base foram registradas, incrementandose um log para posterior análise. Essas etapas reforçam a mais importante das responsabilidades em um website, a gestão do conteúdo (BROWN, 2003).

É possível observar a adequação da estrutura criada ao disposto na literatura de apoio, de modo especial em relação aos elementos básicos para a criação de estruturas digitais, nas quais são priorizadas a organização descritiva, temática, representacional, visual e navegacional de informações (ROSENFELD; MORVILLE, 2002). Esses elementos se referem: aos sistemas de organização, responsáveis pela estruturação e formas de agrupamentos do conteúdo do site; aos processos de rotulagem, responsáveis pela denominação do conteúdo do grupo informacional, agindo na representação ou identificação de conteúdos específicos; e aos sistemas de navegação, responsáveis pela apresentação de pontos de referência ao usuário, por meio de barras de navegação e mapas do site aos sistemas de busca, responsáveis pelo auxílio ao usuário nas consultas.

Resta salientar que cada um dos elementos do sistema tem sido alvo de estudos específicos e considerados parcialmente para maior aprofundamento das especificações. Entretanto, com este relato, pretendeu-se oferecer aos pesquisadores um retrato do que até aqui se 
construiu e dos marcos constitutivos da trajetória da pesquisa mais ampla que tem se construído desde a fase de planejamento da Brapci.

\section{Considerações}

A base de dados Brapci, em menos de dois anos de acesso público pela Internet, vem se consolidando como importante fonte de informação na área de $\mathrm{CI}$, disponibilizando trinta publicações vigentes e históricas. Seleciona e reúne grande parte da literatura pertinente e relevante da especialidade, com critérios de inserção para indexação do corpus a ser incorporado.

A construção da metodologia Brapci foi iniciada com um estudo exploratório que possibilitou a coleta de informações para o diagnóstico detalhado das necessidades relacionadas à implantação de um ambiente que possibilitasse a construção e manutenção de uma base de dados temática. Discussões dentro do grupo de pesquisa contribuíram e foram fundamentais para o diagnóstico e a posterior validação da metodologia de construção da base, como referência para a área de CI.

A metodologia definiu variáveis orientadoras em pesquisa de campo por meio de estudo exploratório com o universo de pesquisadores participantes da lista de discussão da Associação Nacional de Pesquisa e Pós-Graduação em Ciência da Informação (Ancib), possibilitando a definição de algumas demandas dos pesquisadores.

Com a identificação dos requisitos para o sistema, foi possível a concepção do modelo a ser utilizado na metodologia de construção da base de dados. Todas as etapas do processo foram acompanhadas paralelamente por discussões entre os integrantes do Grupo de Pesquisa, com indicações de pontos vulneráveis e contribuições, o que favoreceu as modificações no produto e na metodologia.

Em sua segunda fase, o projeto construiu um produto funcional do sistema, partindo dos requisitos identificados na pesquisa de campo da fase anterior com planejamento do conteúdo textual, classificação e hierarquização das sessões, elaboração do projeto visual bem como a tipologia, iconografia e padronização gráfica a ser utilizada no sistema.

Das opções de linguagens e ferramentas operacionais apropriadas para a execução do produto foram adotados dois critérios, o acesso livre e a adequação à infraestrutura e diretrizes da UFPR.

Com o produto implantado, foi realizada sua validação com uma pesquisa de campo instrumentalizada dentro do grupo de foco composto por pesquisadores da área e indexadores da base, cujo propósito foi um estudo comparativo da metodologia apresentada em contrapartida à utilizada anteriormente no ProCite 5. O produto demonstrou-se, em sua interface, mais amigável ao usuário indexador e a implantação em um ambiente virtual solucionou uma das principais barreiras da metodologia anterior, a qual não possibilitava manutenção remota nem tampouco uso simultâneo de mais de um usuário, podendo apenas um indexador por vez trabalhar na base. 
Com a indexação dos artigos em execução foi possível sua disponibilização para consulta à base de dados pela Internet. Essa disponibilização das fontes de informações referenciais mostrou fragilidade no mecanismo de busca, pois, quando se utilizava a lógica booleana, os usuários reportavam dificuldade na recuperação das informações, principalmente pela impossibilidade do mecanismo recuperar termos compostos. Com base no retorno dos usuários, o grupo de estudo está implantando um novo mecanismo de busca que inclua essa opção, além da recuperação por autores, palavras-chave e títulos de forma simplificada ou avançada.

Uma facilidade da nova metodologia é o monitoramento periódico de novas edições das publicações com a utilização do protocolo de arquivos abertos OAI-PMH, que possibilita a coleta de novos trabalhos publicados, indicando aos indexadores novos artigos. Assim, dispensam-se as visitas periódicas aos sites das publicações. De todas as revistas vigentes somente uma não utiliza esse protocolo para disponibilização de seu conteúdo.

Outro problema constatado, quando da disponibilização on-line, foi a volatilidade dos links que apontavam para o conteúdo completo em algumas fontes de informação. Como o processo de indexação é realizado apenas uma vez, esses links podem ficar desatualizados, principalmente quando a mantenedora da publicação sofre migração de versões dos sistemas ou pela simples descontinuidade da publicação. $O$ grande número de "links quebrados" requer dos indexadores constante acompanhamento e monitoramento dos retornos dos usuários que possibilite a correção dos endereços. A indicação do link incorreto impossibilita ao pesquisador a recuperação direta da fonte, reduzindo a precisão das informações.

Uma proposta de aperfeiçoamento para a metodologia da Brapci, agregando a ela as condições de repositório, seria a incorporação dos artigos completos com a indicação e a manutenção do link da fonte original. A viabilidade dessa proposta provém, principalmente, do fato de que todas as publicações vigentes utilizam a política de arquivos abertos e disponibilização do conteúdo na íntegra, sem restrições. Entretanto, para que o mesmo possa ocorrer em relação às publicações históricas (descontinuadas), serão necessárias medidas relacionadas aos direitos autorais.

Todos os procedimentos aqui relatados foram planejados e executados em processo coletivo de aprendizagem, em atividades promovidas pelo Grupo de Pesquisa Educação, Pesquisa e Perfil Profissional em Informação (E3PI), com envolvimento dos professores e estudantes de graduação e pós-graduação. Reforça-se, desse modo, o caráter pedagógico e o valor do projeto, assim como de seus desdobramentos em atividades de extensão, com propósitos de manutenção, estudos e aperfeiçoamento da Brapci. 


\section{Referências}

BROWN, P. Information architecture with XML: a management strategy. West Sussex: John Wiley, 2003.

BUFREM, L. S. Práticas de organização e divulgação da produção intelectual em ciência da informação no Brasil. Encontros Bibli (UFSC), v. esp., p. 36-53, 2008.

Revistas científicas: características, funções e critérios de qualidade. In: POBLACIÓN, D. A.; WITTER, G. P.; SILVA, J. F. M. (Orgs.). Comunicação e produção científica: contexto, indicadores e avaliação. São Paulo: Angellara, 2006. p. 191-214.

CAMARGO, L. S. A.; VIDOTTI, S. A. B. G. Arquitetura da informação para biblioteca digital personalizável. Encontros Bibli: Revista Eletrônica de Biblioteconomia e Ciência da Informação, Florianópolis, v. 11, n. esp., p. 103-118, 2006.

CENDÓN, B. V. Serviços de indexação e resumo. In: CAMPELLO, B. S.; CENDÓN, B. V.; KREMER, J. M. (Orgs.) Fontes de informação para pesquisadores e profissionais. Belo Horizonte: UFMG, 2000. p. 217-248.

COSTA, F. D. O. Concepção e implantação de protótipo para gerenciamento e disponibilização da Brapci na web. In: EVENTO NACIONAL DE INICIAÇÃO CIENTÍFICA, 17. Anais... Curitiba: UFPR, 2009.

DIAS, E. W. Obras de referência. In: CAMPELLO, B. S.; CENDÓN, B. V.; KREMER, J. M. (Orgs.). Fontes de informação para pesquisadores e profissionais. Belo Horizonte: UFMG, 2000. p. 199-216.

DÜRSTELER, J. Information visualization, what is it all about? Inf@Vis! The digital magazine of InfoVis.net, n. 100, 2002. Disponível em: $<$ http://www.infovis.net/printMag.php?num=100\&lang=2>. Acesso em: 22 ago. 2006.

FOX, C. Making IA real: an overview of an Information Architecture Strategy. In: THE INTERNET CONFERENCE \& EXHIBITION FOR LIBRARIANS \& INFORMATION MANAGERS, Pasadena, CA, Nov 6th 2001. Proceedings... Internet Librarian, 2001. Disponível em: <http://www.onlineinc.com/il2001/tuesday.htm>. Acesso em: 25 set. 2007.

GARCÍA LÓPEZ, G. L. Los sistemas automatizados de acceso a la información bibliográfica: evaluación y tendencias en la era de Internet. Salamanca: Ediciones Universidad de Salamanca, 2007.

GUTWIN, C.; FEDAK, C. Interacting with big interfaces on small screens: a comparison of fisheye, zoom, and panning techniques. Graphics Interface, London, Ontario, n. 49, p. 145-152, 2004

HYLTON, J. Usable information designs. D-Lib Magazine, Reston, Virginia, v. 6, n. 6, jun. 2000. Disponível em: <http://www.dlib.org/dlib/june00/06bookreview.html>. Acesso em: 26 set. 2007. 
KRUEGER, R. A.; CASEY, M. A. Focus groups: a practical guide for applied research. 3. ed. Thousand Oaks: Sage, 2000.

LADRIÈRE, J. Filosofia e práxis cientifica. Rio de Janeiro: F. Alves, 1978.

MEADOWS, A. J. A comunicação científica. Brasília: Briquet de Lemos, 1999.

MUELLER, S. P. M. A comunicação científica e o movimento de acesso livre ao conhecimento. Ciência da Informação, Brasília, v. 35, n. 2, p. 27-38, 2006.

NIELSEN, J. Usability engineering. San Diego: Morgan Kaufmann, 1993.

ROSENFELD, L.; MORVILLE, P. Information architecture for the World Wide Web. 2. ed. Sebastopol, CA: O'Reilly, 2002.

ROZENFELD, H. et al. Gestão de desenvolvimento de produtos: uma referência para a melhoria do processo. São Paulo: Saraiva, 2006.

SANTOS, R. L. G. Usabilidade de interfaces para sistemas de recuperação de informação na web: estudo de caso de bibliotecas on-line de universidades federais brasileiras. 2006. Tese (Doutorado em Design) Programa de Pós-graduação em Design, Pontifícia Universidade Católica do Rio de Janeiro, Rio de Janeiro, 2006.

SAYÃO, L. F. Bases de dados: a metáfora da memória científica. Ciência da Informação, Brasília, v. 25, n. 3, p. 314-318, 1996.

SILBERSCHATZ, A., KORTH, H. F., SUDARSHAN S. Sistema de Banco de Dados. Rio de Janeiro: Campus, 2006.

UNGER, R. J. G.; FREIRE, I. M. Sistemas de informação e linguagens documentárias no contexto dos regimes de informação: um exercício conceitual. Revista Digital de Biblioteconomia \& Ciência da Informação, Campinas, v. 4, n. 1, p. 102-115, jul./dez. 2006.

YU, H. Content and workflow management for library websites: case studies. California, USA: Information Science Publishing, 2005. p. 26-27. 